\title{
15. Internationale Metallographie-Tagung
}

\author{
Helmut Clemens, Svea Mayer und Michael Panzenböck
}

Lehrstuhl für Metallkunde und metallische Werkstoffe, Department Werkstoffwissenschaft, Montanuniversität Leoben, Leoben, Österreich

Online publiziert 27. März 2019

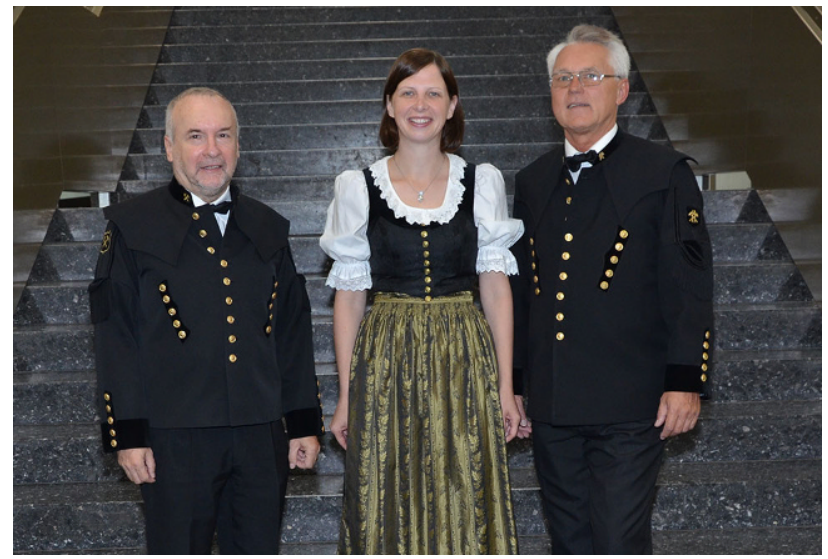

Vom 19.-21. September 2018 fand die vom Lehrstuhl Metallkunde und metallische Werkstoffe organisierte 15. Internationale Metallographie-Tagung an der Montanuniversität Leoben statt. Insgesamt haben an der von Prof. Dr. Helmut Clemens, assoz. Prof. Dr. Svea Mayer und Dr. Michael Panzenböck veranstalteten Konferenz ca. 400 Personen aus 10 Ländern teilgenommen.

In 71 Vorträgen, davon sieben Plenarvorträgen, wurden die aktuellen Erkenntnisse auf dem Gebiet der Materialographie präsentiert und diskutiert. Die Fachgebiete erstreckten sich von Präparationstechnik und Gefügecharakterisierung bis hin zu Tomographie und 3D-Auswerte- und Analysemethoden. In den Plenarvorträgen wurden die kommenden Herausforderungen an das Fachgebiet hervorgehoben, wie „Big Data" und „Machine Learning,'Hochdurchsatzcharakterisierung sowie die immer stärker werdende Elektromobilität.
In einer parallel laufenden Geräteausstellung haben 39 Firmen ihre Produkte und Dienstleistungen präsentiert. Im Rahmen der Veranstaltung wurde der Roland-MitschePreis an Prof. Dr. Andreas Neidel, Siemens AG, für seine herausragenden Leistungen auf dem Gebiet der Schadensanalyse und Metallographie verliehen. Mit den BuehlerPreisen wurden Autorinnen und Autoren für ihre Artikel in der Zeitschrift Praktische Metallographie (Practical Metallography) ausgezeichnet.

Am letzten Tag der Veranstaltung fand ein spezieller Vortragsblock zur Würdigung des verstorbenen Ehrenvorsitzenden des Fachausschusses Materialographie, em. O. Univ.-Prof. Dr. Dr. h. c. Franz Jeglitsch, statt.

Die vorliegende Ausgabe enthält sieben ausgewählte Beiträge der 15. Internationalen Metallographie-Tagung, die einen Überblick über die oben angeführten Themenschwerpunkte geben. Die Gastherausgeber dieses Heftes wünschen den Leserinnen und Lesern eine spannende Lektüre und hoffen Sie bei der 16 . Internationalen Metallographie-Tagung im September 2022 in Leoben begrüßen zu dürfen.

Ein herzliches Glück Auf,

Helmut Clemens, Svea Mayer und Michael Panzenböck

\footnotetext{
$\overline{\text { Prof. Dr. H. Clemens }(\bowtie)}$

Lehrstuhl für Metallkunde und metallische Werkstoffe,

Department Werkstoffwissenschaft,

Montanuniversität Leoben,

Roseggerstraße 12,

8700 Leoben, Österreich

helmut.clemens@unileoben.ac.at
} 